\title{
MEGAFAUNA PLEISTOCÊNICA DA MICRORREGIÃO DE SENHOR DO BONFIM, BAHIA
}

\author{
Márcia Cristina Teles Xavier ${ }^{1}$ \\ Mário André Trindade Dantas ${ }^{2}$ \\ 10.18190/1980-8208/estudosgeologicos.v28n2p19-31 \\ Cristiana Cerqueira da Silva-Santana ${ }^{3}$ \\ ${ }^{1}$ Doutoranda no Programa em Ecologia e Conservação, UFSE, mxavih@yahoo.com.br \\ ${ }^{2}$ Laboratório de Ecologia e Geociências, Campus Anísio Teixeira - Instituto Multidisciplinar \\ em Saúde, UFBA, matdantas@yahoo.com.br \\ ${ }^{3}$ Laboratório de Arqueologia e Paleontologia, Departamento de Educação Campus VII UEBA, \\ ccsilva@uneb.br
}

\section{RESUMO}

A ocorrência de mamíferos do Pleistoceno é registrada desde o século XIX em quase todos os estados do Brasil. Na Bahia, entre 1817 e 1820, Johann Baptiste von Spix e Carl von Martius relataram fósseis no povoado de Barriga Mole (Andorinha), microrregião de Senhor do Bonfim, mas somente a partir da década de 1980 esta área começou a figurar na bibliografia nacional, especialmente após os trabalhos de campo de Castor Cartelle (PUC-MG) e colaboradores. A coleção do Laboratório de Arqueologia e Paleontologia da UNEB (Campus VII) apresenta fósseis de mamíferos do Pleistoceno da microrregião de Senhor do Bonfim, coletados em cavernas e em outros jazigos fossilíferos de quatro municípios: Senhor do Bonfim, Campo Formoso, Filadélfia e Andorinha. No presente trabalho foram estudados e identificados seis taxa de mamíferos, incluindo Eremotherium laurillardi, Glyptotherium sp., Panochthus sp., Notiomastodon platensis, Palaeolama major e Toxodontinae indeterminado, colaborando com o conhecimento acerca da composição paleofaunística da região durante o Pleistoceno final.

Palavras chave: Megamamíferos, Paleovertebrados, Quaternário Tardio, Paleoecologia, Dieta.

\begin{abstract}
Occurrence of Pleistocene mammals in Brazil is recorded since nineteenth century in almost all states of Brazil. In Bahia, between 1817 and 1820, Johann Baptiste von Spix and Carl von Martius reported fossils in Barriga Mole (Andorinha), integral area of Senhor do Bonfim, but only in the 1980's, the area will compose the repeatedly bibliography national and paleontological world, especially after field works of Castor Cartelle (PUC-MG) and collaborators. The collection of the Laboratory of Archeology and Paleontology of the UNEB (Campus VII) presents fossils of Pleistocene mammals of the micro-region of Senhor do Bonfim collected in caves and other fossiliferous deposits of four municipalities: Senhor do Bonfim, Campo Formoso, Filadélfia e Andorinha. In the present work six taxa of mammals, including Eremotherium laurillardi, Glyptotherium sp., Panochthus sp., Notiomastodon platensis, Palaeolama major and Toxodontinae indeterminate were studied and identified, collaborating with the knowledge about the paleofaunistic composition of the region during the Late Pleistocene.
\end{abstract}

Keywords: Megamammals, Paleovertebrates, Late Quaternary, Paleoecology, Diet. 


\section{INTRODUÇÃOO}

Os mamíferos fósseis pleistocênicos compreendem animais classificados como gigantes (massa corpórea $>1000 \mathrm{Kg}$ ), grande porte (100$1000 \mathrm{~kg}$ ), médio porte $(10-100 \mathrm{~kg}) \mathrm{e}$ pequeno porte $(<10 \mathrm{Kg})$, que inclui em sua maioria taxa viventes (Prevosti \& Vizcaíno, 2006). No Brasil, estão representados por Ordens como Cingulata, Notoungulata, Proboscidea e Carnivora (e.g. Cartelle, 1999), comumente encontrados em cavernas (e.g. Auler \& Smart 2001), depósitos de tanques (e.g. Cartelle, 1995; Dantas et al, 2013a) e alguns depósitos fluviais da Amazônia (e.g. Holanda \& Cozzuol, 2006).

A ocorrência da megafauna pleistocênica no Brasil é registrada desde o século XIX, em quase todos os Estados do Brasil (Paula Couto, 1979). Entre 1817 e 1820 Johann Baptiste von Spix e Carl von Martius relataram achados fósseis na microrregião de Senhor do Bonfim, no Povoado de Barriga Mole, município de Andorinha-BA (Spix \& Martius, 1976), mas somente a partir da década de 1980, que a microrregião passou a figurar na bibliografia paleontológica nacional e mundial com maior intensidade.

Na Bahia, Cartelle (1993) coletou um molar superior e diversas peças esqueletais na Toca da Boa Vista em associação com restos de Tayassu peccari (Fisher), Mazama sp., Smilodon populator Lund e Lama guanicoe (Muller). Dantas et al. (2013b), estudaram Notiomastodon platensis em Jaguarari, datando um molariforme pela técnica de Ressonância de Spin Electrônico - RSE situando a sua ocorrência por volta de $114 \pm 20 \mathrm{ka}$.
Deste modo, o presente trabalho tem como objetivos: (i) registrar a ocorrência de fósseis de seis táxons da megafauna pleistocênica encontrados em quatro municípios da microrregião de Senhor do Bonfim; e (ii) discutir a paleoecologia destes táxons.

\section{MATERIAIS E MÉTODOS}

\section{Área de estudo}

O Território de Identidade Piemonte Norte do Itapicuru está localizado no Centro Norte Baiano, entre as coordenadas aproximadas de $9^{\circ} 47^{\prime}$ a $11^{\circ} 9^{\prime}$ de latitude sul e $39^{\circ} 44^{\prime}$ a $41^{\circ} 16^{\prime}$ de longitude oeste, ocupando uma área de $14.123 \quad \mathrm{~km}^{2} \quad$ (IBGE, 2013). Administrativamente é composto pelos municípios de Andorinha, Antônio Gonçalves, Caldeirão Grande, Campo Formoso, Filadélfia, Jaguarari, Pindobaçu, Ponto Novo e Senhor do Bonfim (SEI, 2015; Fig. 1).

Todos os municípios estão inseridos na Região Semiárida com ocorrência também de uma faixa árida no município de Campo Formoso. Entre Senhor do Bonfim e Pindobaçu, há influência do clima subúmido a seco, com chuvas de outono/inverno e primavera/verão (SEI, 1998).

A vegetação do território é formada por Caatinga Arbórea e Arbustiva, Cerrado, contato CerradoFloresta Estacional, vegetação secundária e contato Cerrado-Caatinga. Entre Senhor do Bonfim e Caldeirão Grande há áreas de Tensão Ecológica. O Refúgio Ecológico Montano, em Pindobaçu e Antônio Gonçalves (Serra de Jacobina), e os Campos Rupestres em Campo Formoso (Serras Setentrionais), completam a paisagem vegetacional (SEI, 2018). 


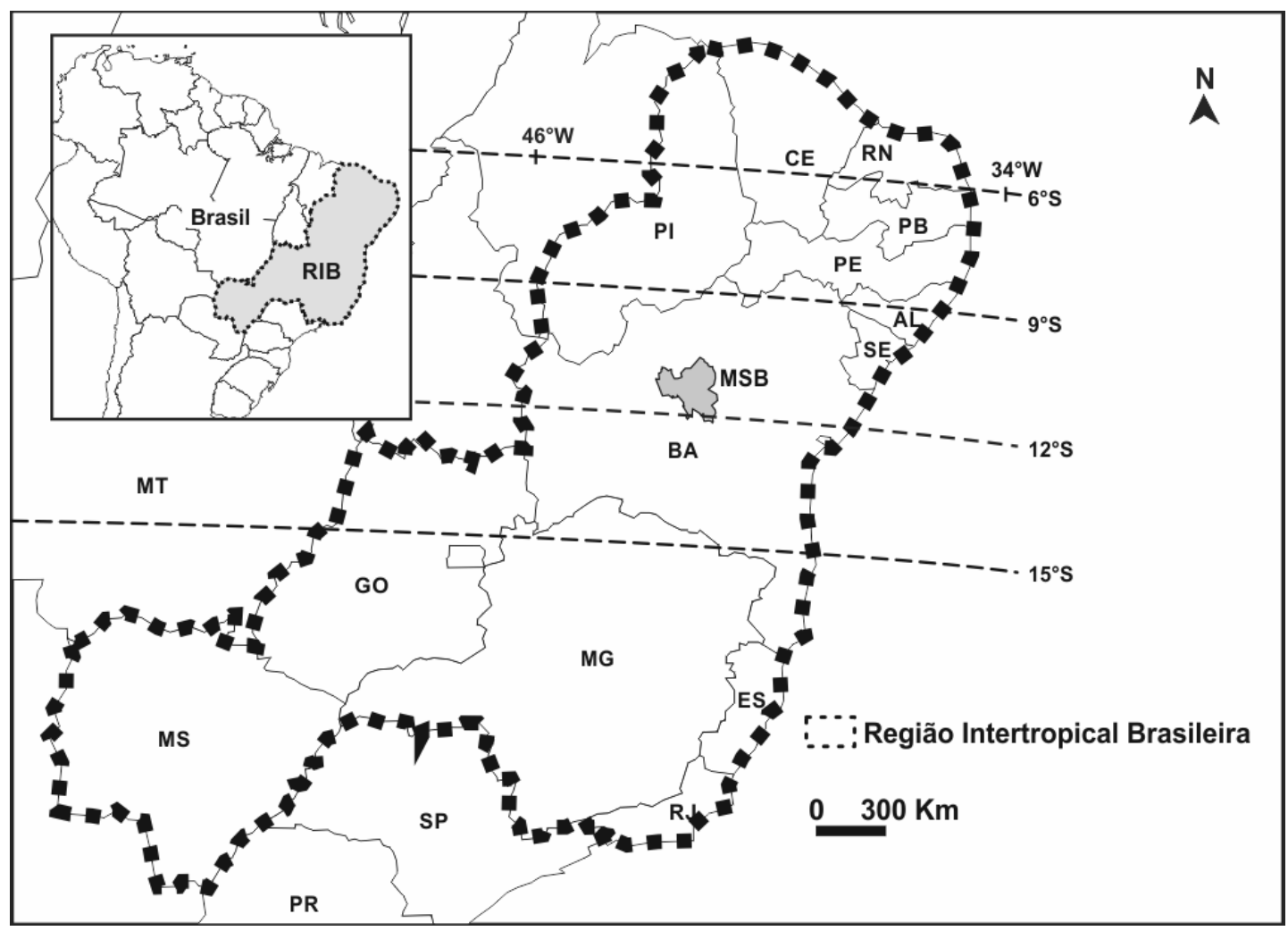

Figura 1 - Mapa de localização da microrregião de Senhor do Bonfim (MSB). A linha tracejada indica a Região Intertropical Brasileira. Adaptado de Dantas, 2017.

\section{Preparação e identificação do material fóssil coletado}

O material foi coletado pelos moradores em cavernas na microrregião, ou achados quando ocorria a abertura e/ou aprofundamento de tanques e também durante obras de engenharia.

As primeiras doações de fósseis ao Laboratório de Arqueologia e Paleontologia (LAP), foram realizadas por professores, alunos e moradores de cada região. A ampliação da coleção foi feita em algumas etapas de campo, com coletas sistematizadas, na tentativa de não só resgatar o material fóssil, mas coletar informações tafonômicas que auxiliem a melhor interpretação das assembleias.

Em laboratório, os fósseis foram limpos, lavados em água corrente, com o auxílio de uma escova pequena para extrair o excesso de sedimento. Após a lavagem, os fósseis foram expostos em cima das bancadas sobre papel, à sombra para secar. Depois de secos, os fósseis foram restaurados com cola para isopor.

$\mathrm{O}$ procedimento seguinte consistiu em separar os ossos por localidade de coleta e por tipos anatômicos, e então, identificá-los e descrevê-los. Todos os fósseis identificados foram numerados com caneta nanquim e tombados no Laboratório de Arqueologia e Paleontologia.

Os fósseis descritos nesse trabalho foram coletados em Senhor do Bonfim, Filadélfia, Campo Formoso e Andorinha. Constam de elementos mandibulares e algumas peças bastante fragmentadas do esqueleto pós-craniano que apresentam diferentes tonalidades de coloração. Essa diferença sugere variações nos padrões mineralógicos, impregnação sofrida pelo material e tempo de exposição na superfície do tanque e nas cavernas.

A sistemática dos Tardigrada segue Cartelle \& De Iuliis (1995); de 
Proboscidea segue Mothé et al. (2011); de Notoungulata segue Miño-Boilini et al. (2006); de Cingulata segue Oliveira et al. (2010); e de Perissodactyla segue Prado \& Alberdi (2008). A descrição anatômica das peças segue, com leves adaptações, Paula Couto (1979).

\section{RESULTADOS E DISCUSSÃO}

\section{Sistemática}

TARDIGRADA Latham \& Davies, 1795

MEGATHERIOIDEA Gray, 1821

MEGATHERIIDAE Owen, 1843

MEGATHERIINAE Gill, 1872

Eremotherium laurillardi (Lund, 1842)

Cartelle \& Bohórquez, 1982

Material. Andorinha: fragmentos de molariformes LAP 050, 051, 052, 053; corpo de vértebra LAP 036; corpo de vértebra torácica LAP 034; corpo de vértebras lombares LAP 029, 032, 033; corpo de vértebras caudais LAP 030, 035; corpo de vértebras caudais LAP 042; estérnebra LAP 038; porção proximal de metacarpo III LAP 057; unciforme LAP 037; magno LAP 039. Campo Formoso: Parte do ramo mandibular esquerdo LAP 014; corpo de vértebra lombar LAP 019; ulna? LAP 009; porção distal da tíbia esquerda LAP 011. Filadélfia: astrágalos esquerdos LAP 015. Senhor do Bonfim: corpo do axis LAP 028; estérnebra LAP 026, metacarpo IV LAP 025.

Comentários. A Eremotherium laurillardi são atribuídos materiais cranianos e pós-cranianos diversos. Dos materiais cranianos, mais precisos para a diagnose específica, estão presentes parte do ramo mandibular esquerdo LAP 014, onde se observa a série de molariformes do $\mathrm{M}_{2}$ ao $\mathrm{M}_{4}$ (Fig. 2A-B), além de diversos molariformes isolados. Como característico do gênero, os molariformes são prismáticos e hipsodontes (sem raízes, de crescimento continuo; Paula Couto, 1979).

A preguiça terrestre E. laurillardi foi uma espécie de hábitos herbívoros, apresentando caracteres mais primitivos que as espécies do gênero Megatherium. A mandíbula apresenta séries dentárias mais afastadas entre si, e contorno interno dos molariformes convexo, além da bossa inferior do dentário com contornos mais suaves (Paula Couto, 1979), características observadas no fóssil LAP 014 (Fig. 2A-B).

Apesar de proposta alternativa (e.g. Guérin \& Faure, 2000), é provável que E. laurillardi constitua uma única espécie panamericana, ocorrendo desde o sul dos Estados Unidos até a Argentina, e em quase todos os Estados do Brasil (Cartelle \& Bohorquez, 1982; Cartelle \& De Iuliis, 1995; 2006), sendo esta portanto a classificação adotada no presente trabalho.

Eremotherium

laurillardi

possivelmente tinha preferências climáticas por ambientes tropicais e subtropicais, o que pode ser inferido pelo seu registro limitado nos Estados Unidos, indicando provavelmente, o limite norte de sua distribuição (Mcdonald \& Lundelius, 2009). 

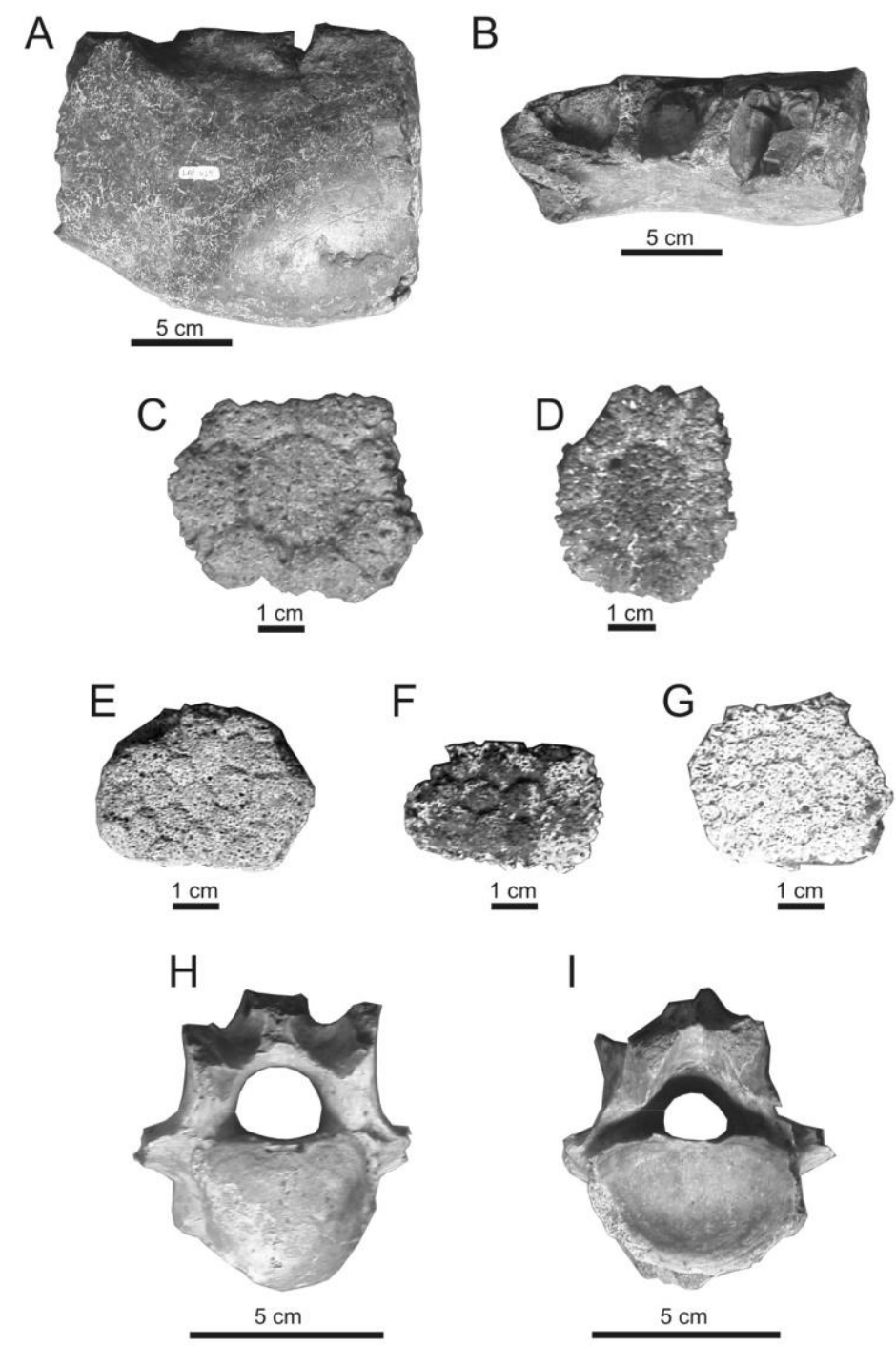

Figura 2 - Eremotherium laurillardi: ramo mandibular esquerdo LAP 014, (A) vista lingual, (B) vista oclusal; Glyptotherium sp.: osteodermos de carapaça LAP 048 (C) e LAP 054 (D); Panochthus sp.: osteodermos de carapaça LAP 046 (A); LAP 047 (B); LAP 045 (C); Palaeolama major: vértebra cervical LAP 041, (A) vista dorsal, (B) vista ventral.

CINGULATA Illiger, 1811

GLYPTODONTOIDEA Simpson, 1931

GLYPTODONTIDAE Burmeister, 1879

GLYPTODONTINAE Trouessart, 1898

Glyptotherium sp.

Material. Andorinha: osteodermos isolados, LAP 048, 049, 054, 055 e 056.
Comentários. Em revisão dos Glyptodontinae, Oliveira et al. (2010), sugerem a presença do gênero norteamericano Glyptotherium sp. para todo o Nordeste e Sudeste do Brasil. Deste modo, a este gênero são atribuídos cinco osteodermos, em bom estado de conservação, podendo ser observado o padrão típico em roseta que constitui a ornamentação externa, delimitada por sulcos mais rasos do que em Glypodon, que em parte caracteriza as espécies do 
gênero Glyptotherium (Oliveira et al., 2010). Estas características estão bastante visíveis nos osteodermos LAP 048 e 054 (Fig. 2C-D), no qual se observa uma figura central de maior tamanho, circundada por figuras periféricas poligonais de menor tamanho que esta última, podendo ocorrer em número de sete a treze nesta espécie.

As espécies do gênero Glyptotherium seriam animais herbívoros, que provavelmente alimentavam-se de gramíneas, sendo considerados por alguns autores como pastadores restritos (grazer; Rincon et al., 2008). No Nordeste brasileiro, fósseis deste gênero já foram encontradas nos Estados da Bahia, Sergipe, Pernambuco, Paraíba e Rio Grande do Norte (Porpino et al., 2004; Dantas et al., 2005; Silva et al., 2006; Andrade et al.,2017 ).

HOPLOPHORINAE Weber, 1925

PANOCHTINI Simpson, 1945

\section{Panochthus sp.}

Material. Andorinha: Osteodermos isolados, LAP 045, 046 e 047.

Comentários. Ao gênero Panochthus sp. atribui-se três osteodermos, onde LAP 046 e 047 (Fig. 2E-F) encontram-se em bom estado de conservação, apresentam como característica em sua superfície externa um aglomerado de pequenas figuras, de mesmo tamanho, separadas entre si por sulcos estreitos e rasos.

O osteodermo LAP 046 apresenta formato hexagonal e o LAP 047 apresenta formato retangular, a parte interna de ambos se apresenta lisa, e os dois possuem espessura que varia entre $17 \mathrm{~mm}$ a $22 \mathrm{~mm}$.

O osteodermo LAP 045 (Fig. 2G), encontra-se em bom estado de conservação, e uma figura central de forma circular com tamanho de $11 \mathrm{~mm}$, rodeada por duas fileiras de figurinhas periféricas, e que apresentam tamanho de $6 \mathrm{~mm}$.

O gênero Panochthus, é o mais conhecido da tribo Panochtini Simpson, 1945, distribuindo-se cronologicamente por todo o Pleistoceno. $\mathrm{O}$ grupo compreende espécies de grande tamanho (Paula-Couto, 1979), hábitos herbívoros, provavelmente pastadores de gramíneas (Zurita et al., 2005).

No Nordeste brasileiro este gênero já foi registrado nos Estados da Bahia, Pernambuco, Paraíba, Rio Grande do Norte e Piauí (Guerin et al., 1993; Bergqvist et al., 1997; Porpino \& Bergqvist, 2002; Porpino et al., 2004; Silva et al., 2006).

\section{ARTIODACTYLA Owen, 1848 TYLOPODA Illiger, 1811 CAMELIDAE Gray, 1821 CAMELINAE Zittel, 1893}

\section{Palaeolama major Liais, 1872}

Material. Andorinha: vértebra cervical, LAP 041.

Comentários. À espécie Palaeolama major é atribuída uma vértebra cervical LAP 041(Fig. 2H-I) em bom estado de conservação, apresentando medidas e características semelhantes às vértebras descritas por Hoffstetter (1952), como por exemplo, face articular anterior ligeiramente convexa, e posterior côncava.

Espécimes de Palaeolama major já foram encontrados em cavernas de Minas Gerais e da Bahia (Paula Couto, 1979), além de ocorrências em tanques nos Estados de Sergipe, Pernambuco, Paraíba, Rio Grande do Norte, Ceara e Piauí (Guerin et al., 1993; Bergqvist et al., 1997; Porpino et al., 2004; Dantas et al., 2005; Silva et al., 2006).

PROBOSCIDEA Illiger, 1811 ELEPHANTOIDEA Gray, 1821 
GOMPHOTHERIIDAE Hay, 1922

Notiomastodon platensis (Ameghino, 1888)

Material. Andorinha: fragmento de molar, LAP 024. Campo Formoso: vértebra cervical, LAP 012. Filadélfia: fragmento de molar, LAP 006.

Comentários. Os materiais estudados estão ilustrados na Figura 3A-D.

Em sua revisão dos gonfotérios Sul Americanos Mothé et al. (2011) sugerem para este táxon uma nova combinação, sugerindo o binômio Notiomastodon platensis (Ameghino, 1888). Desta forma, há uma redução do número de espécies dos gonfotérios na América do Sul, passando para dois gêneros e duas espécies, apresentando $N$. platensis distribuição geográfica no Brasil, Equador, Venezuela, Colômbia, Peru, Paraguai, Chile, Argentina, e Uruguai.

Fósseis de Notiomastodon platensis são encontradas em quase todos os Estados do Nordeste do Brasil, geralmente em associação com $E$. laurillardi. É uma espécie herbívora, que apresentava alimentação mista, com tendência pastadora (Gadens-Marcon, 2008).

NOTOUNGULATA Roth, 1903

TOXODONTIA Owen, 1853

TOXODONTIDAE Owen, 1845

TOXODONTINAE Trouessart, 1898

Material. Senhor do Bonfim: tíbia esquerda, LAP 007.

Descrição e Comentários. A tíbia apresenta um bom estado de conservação, estando ausente apenas a sua porção proximal (Fig. 3E-G).

Esta ordem é a mais diversificada dentro dos ungulados nativos (Bond, 1986, 1999; Bond et al., 1995). Ecologicamente estavam relacionados com ambientes abertos, cercados de corpos d'água e hábitos pastadores (Oliveira, 1999; Czerwonogora et al., 2003), possuindo uma dieta com preferência por gramíneas abrasivas (Macfadden, 2005), com crescimento contínuo dos dentes (Cartelle, 1994). Estudos recentes apontam a presença de duas espécies de Toxodontíneos para o Nordeste do Brasil: Toxodon platensis Owen, 1837 e Piauhytherium capivarae (Guerin \& Faure, 2013). 

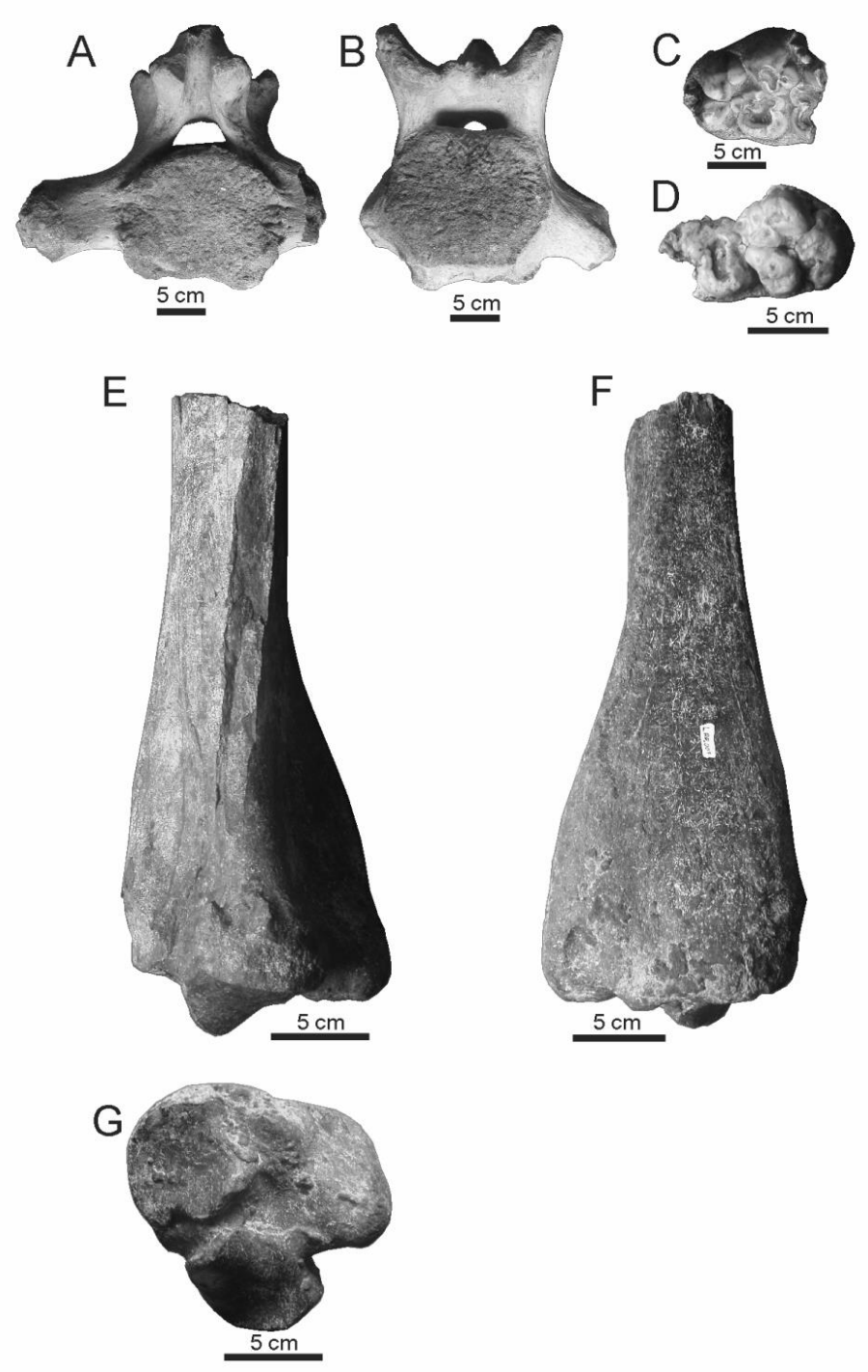

Figura 3 - Notiomastodon platensis: vértebra cervical, LAP 012, (A) vista dorsal, (B) vista ventral; molariforme, LAP 024, (C) vista oclusal; molariforme, LAP 026, (D) vista oclusal; Toxodontinae: tíbia, LAP 007, (E) vista dorsal, (F) vista ventral; (G) vista posterior.

\section{Interpretações Paleoecológicas}

As identificações taxonômicas realizadas indicam a presença de seis taxa de mamíferos pleistocênicos na região estudada, incluindo Eremotherium laurillardi, Glyptotherium sp., Panochthus sp., Notiomastodon platensis, Palaeolama major e Toxodontinae.

De um modo geral, os fósseis se encontrem fragmentados devido a fatores tafonômicos (não abordados neste trabalho) e ao tipo de coleta não sistemática realizada nesses sítios. É importante salientar que mesmo as coletas efetuadas pela equipe do LAP, foram realizadas nas áreas de refugo dos tanques, o que proporcionou coletar material já descontextualizado pela ação das escavações feitas pelos moradores da zona rural.

Para a autoecologia da megafauna pleistocênica encontrada no Nordeste do Brasil, atualmente sugere-se que Eremotherium laurillardi, Notiomastodon platensis e Toxodontinae apresentavam alimentação mista de 
plantas $\mathrm{C}_{3}$ e $\mathrm{C}_{4}$, com ampla largura de nicho (Dantas et al., 2017). Tanto $N$. platensis quanto Toxodontinae seriam animais de grande tamanho e hábitos pastadores ou pastadores/ramoneadores (Macfadden, 2005; Prado et al., 2005; Dantas et al., 2013a) e ocorreriam em regiões semelhantes.

E. laurillardi e Toxodontinae são associados a um habitat do tipo savana (com áreas recobertas por gramíneas) em associação com fisionomias mais fechadas (borda de floresta). Este fato implica em um clima mais úmido do que o atual em certos momentos do Pleistoceno final (Araújo Júnior \& Porpino, 2007; Ribeiro \& Carvalho, 2009). Estas condições ambientais estariam de acordo com o modelo para o Pleistoceno do Brasil Intertropical ExtraAmazônico proposto por Cartelle (1999).

Panochthus sp. habitava as savanas e alimentava-se de gramíneas (Ranzi, 2000) e a presença de Palaeolama major indica que na região estudada o clima durante a transição Pleistoceno-Holoceno era mais frio, com predominância de áreas abertas. O registro de Glyptotherium sp. no final do Pleistoceno do Nordeste do Brasil sugere relações biogeográficas com o norte da América do Sul e Central e Sul da América do Norte.

Em virtude a presença de alguns animais, tais como E. laurillardi e Panochthus sp., estes podem ser localizados no NeopleistocenoHoloceno (Porpino et al., 2004), equivalente a Idade Lujanense (Cione \& Tonni, 1999).

\section{CONSIDERAÇÕES FINAIS}

A partir deste conjunto fossilífero analizado, sugere-se condições ambientais diversas da encontrada atualmente para a região, provavelmente semelhantes àquelas inferidas por Cartelle (1999) para o Pleistoceno do Brasil Intertropical Extra-Amazônico, onde, durante o Pleistoceno final Holoceno, a região compreenderia áreas abertas de savana associadas, possivelmente, com fisionomias mais fechadas, tipo florestas.

\section{REFERÊNCIAS}

Andrade, Luana Cardoso de; Oliveira, Édison Vicente; Souza, Bartolomeu Israel de. 2017. Novos Achados de Xenarthra em um Depósito de Tanque do Pleistoceno da Paraíba, Nordeste do Brasil. Revista de Geologia, v. 30, n.1.33-46.

Araújo Junior H.I., Porpino K.O. 2007. Mamíferos fósseis da Fazenda Lágea Formosa, São Rafael, Rio Grande do Norte, Brasil: Interpretações paleoecológicas. In: VIII Congresso de Ecologia do Brasil. Boletim de resumos. Anais, p. 1-2.

Auler A.S, Smart P.L. 2001. Late Quaternary paleoclimate in semiarid northeastern Brazil from Useries dating of travertine and water table speleothems. Quaternary Research 55: 159-167.

Bergqvist, Lilian Paglarelli; Gomide, Márcia; Cartelle, Cástor; CAPILLA, R. 1997. Faunas locais e mamíferos pleistocênicos de Itapipoca/Ceará, Taperoa/Paraíba, e Campina Grande/Paraíba. Estudo comparativo, Bioestratinomico e Paleoambiental. Revista Universidade Guarulhos: Geociências, vol. 2, n 6 , p. 23-32. Bond, M. 1986. Los ungulados fósiles de Argentina: evolución y paleoambientes. Actas IV Congreso Argentino Paleontología, Bioestratigrafía, Mendoza, 2, 173-185.

Bond, M. 1999. Quaternary native ungulates of Southern South America. A synthesis. In: Quaternary of South America and 
Antarctic Peninsula (Eds. J. Rabassa \& M. Salemme). Centro Austral de Investigaciones Científicas and Universidad Nacional de la Patagonia, Ushuaia, Tierra de Fuego, 12, 177-205.

Bond, M., Cerdeño, E. P.; López, G. 1995. Los ungulados nativos de América de Sur. In: Evolución climática y biológica de la región Pampeana durante los últimos cinco millones de años. Un ensayo de correlación con el Mediterráneo occidental (Eds. M. T. Alberdi, G. Leone; E. P. Tonni). Monografías del Museo de Ciencias Naturales, Consejo Superior de Investigaciones Científi cas, Madrid, 12, 259-275.

Cartelle, C. 1993. Presença de Lama (Artiocactyla, Camelidae) no Pleistoceno Final-Holoceno da Bahia. In: Congresso Brasileiro de Paleontologia, 13, e Simpósio Paleontológico do Cone Sul, 1, São Leopoldo, 1993. Boletim de Resumos, Rio Grande do Sul, p.139.

Cartelle, C. 1994. Tempo Passado: Mamíferos do Pleistoceno em Minas Gerais. Belo Horizonte: Palco, $131 \mathrm{p}$.

Cartelle, C. 1995. A fauna local de mamíferos pleistocênicos da Toca da Boa Vista (Campo Formoso, BA). Tese para professor titular, Universidade Federal de Minas Gerais.

Cartelle, C. 1999. Pleistocene mammals of the Cerrado and Caatinga of Brazil. In: J. F. Eisenberg ; K. H. Redford (eds.) Mammals of the Neotropics. The Central Neotropics. 3. The University of Chicago Press, p. 27-46.

Cartelle, Cástor ; Bohórquez, German A. 1982. Eremotherium laurillardi (Lund, 1842). Parte I. Determinação específica e dimorfismo sexual. Iheringia Série Geológica, n 7, p. 45-63.

Cartelle, C.; De Iuliis, G. 1995. Eremotherium laurillardi: the Panamerican late Pleistocene Megatheriid sloth. Journal of Vertebrate Paleontology 15(4): 830-841.

Cartelle, Cástor; De Iuliis, Gerard. 2006. Eremotherium laurillardi (Lund) (Xenarthra, Megatheriidae), the Panamerican giant ground sloth: taxonomic aspects of the ontogeny of skull and dentition. Journal of Systematic Paleontology, vol. 4, $\mathrm{n}^{\circ}$ 2, p. 199-209.

Cione, A.L.; Tonni, E.P. 1999. Biostratigraphy and chronological scale of upper-most Cenozoic in the Pampean Area, Argentina. In: J. Rabassa \& M. Salemme (eds.) Quaternary of South America and Antarctic Peninsula, A.A. Balkema Publishers, p. 23-52.

Czerwonogora, A., De EstebanTrivigno, S.; Fariña, R. A. 2003. Hábitos alimentícios de ungulados pleistocenos sudamericanos: un enfoque ecomorfológico. Ameghiniana (Resúmenes), 40, 83R

Dantas, Mário André Trindade.; Zucon, Maria Helena; Ribeiro, Ana Maria. 2005. Megafauna pleistocênica de Gararu, Sergipe, Brasil. Revista de Geociências - UNESP, vol. 24, $\mathrm{n}^{\circ}$ 3. p. 277-287.

Dantas, M.A.T., Dutra, R.P., Cherkinskyc, A., Fortier, D.C., Kaminod, L.H.Y., Cozzuol, M.A., Ribeiro, A.S., Vieira, F.S. 2013a. Paleoecology and radiocarbon dating of the Pleistocene megafauna of the Brazilian Intertropical Region. Quaternary Research 79(1): 61-65.

Dantas, M.A.T., Xavier, M.C.T., França, L.M., Cozzuol, M.A., Ribeiro, A.S.,

Figueiredo, A.M.G., Kinoshita, A., 
Baffa, O., 2013b. A review of the time scale andpotential geographic distribution of Notiomastodon platensis (Ameghino, 1888) in the late Pleistocene of South America. Quat. Int. 317, 73-79.

Dantas, Mário André Trindade; Cherkinsky, Alexander ; Bocherens, Hervé ; Drefahl, Morgana; Bernardes, C.; França, Lucas de Melo, 2017 . Isotopic paleoecology of the Pleistocene megamammals from the Brazilian Intertropical Region: Feeding ecology $(\delta 13 \mathrm{C})$, niche breadth and overlap. Quaternary Science Reviews, v. 170, p. 152-163.

Gadens Marcon, G.T. 2008. Contribuição ao estudo dos Proboscidea (Mammalia, Gomphotheriidae) do Quaternário do Estado do Rio Grande do Sul, Brasil. Revista UnG Geociências, 7(1):93-109.

Guérin, Claude \& Faure, Martine. 2000. La véritable nature de Megatherium laurillardi Lund, 1842 (Mammalia, Xenarthra): un nain parmi les géants. Geobios, vol. $33, n^{\circ} 4$, p. $475-488$.

Guérin C.; Faure M. 2013. - Un nouveau Toxodontidae (Mammalia, Notoungulata) $\mathrm{du}$ Pléistocène supérieur du Nordeste du Brésil. Geodiversitas 35 (1): 155-205.

http://dx.doi.org/10.5252/g2013n1 a7

Guérin, Claude; Curvello, M.A.; Faure, Martine; Hugueney, M.; MourerChauviré, C. 1993. La faune pléistocène du Piauí (Nordeste du Brésil): implications paléoécologiques et biochronologiques. Quaternaria Nova, $n^{\circ} 3$, p. 303-341.

Hoffstetter, R. 1952. Les mammifères pléistocènes de la République de l'Équateur. Mémoires de la Société géologique de France, [S.1.], v. 21, n. 1-4, p.1-391, pis. I-VII.

Holanda, Elizete Celestino; Cozzuol, M. A. . 2006. New records of Tapirus from the Late Pleistocene of Southwestern Amazonia. Revista Brasileira de Paleontologia, v. 9, p. 193-200.

IBGE - Instituto Brasileiro de Geografia e Estatística. 2013. Resolução $n^{\circ} 1$, de 15 de janeiro de 2013. Aprova os valores de áreas territoriais do Brasil, Estados e Municípios. Diário Ofcial da União, Brasília, 23 jan.

Mcdonald, H. Gregory; Lundelius, JR Ernest L. 2009 The giant ground sloth Eremotherium laurillardi (Xenarthra, Megatheriidae) in Texas. Albright, L. B. III, ed., Papers on Geology, Vertebrate Paleontology, and Biostratigraphy in Honor of Michael $\mathrm{O}$. Woodburne. Museum of Northern Arizona Bulletin 65, Flagstaff, Arizona.

MacFadden, B.L. 2005. Diet and habitat of toxodont megaherbivores (Mammalia, Notoungulata) from the late Quaternary of South and Central America. Quaternary Research, 64: 113-124.

Miño Boilini, A.R., Cerdeño, E.; Bond, M. 2006. Revisión del género Toxodon Owen, 1837 (Notoungulata: Toxodontidae) en el Pleistoceno de las provincias de Corrientes, Chaco y Santa Fe, Argentina. Revista Española de Paleontología, 21(2):93-103.

Mothé, D.; Avilla, L.S.; Cozzuol, M.A.; Winck, G.R.. 2011. Taxonomic revision of the Quaternary gomphotheres (Mammalia: Proboscidea: Gomphotheriidae) from the South American lowlands. Quaternary International. doi:10.1016/j.quaint.2011.05.018

Oliveira, E. 1999. Quaternary vertebrate and climates from southern Brazil. 
In: Quaternary of South America and Antarctic Peninsula (Eds. J. Rabassa \& M. Salemme). Centro Austral de Investigaciones Científi cas y Universidad Nacional de La Patagonia, Ushuaia, Tierra de Fuego, 5, 61-75.

Oliveira, E.V.; Porpino, K.; Barreto, A.M.F. 2010. On the presence of Glyptotherium in the late Pleistocene of Northeasthern Brazil, and the status of "Glyptodon" and "Chlamydotherium":

paleobiogeographic implications. Neues Jahrbuch für Geologie und Palaontologie, 258:353-363.

Paula Couto, Carlos de. 1979. Tratado de Paleomastozoologia. Rio de Janeiro: Academia Brasileira de Ciências, 590p.

Porpino, Kleberson de Oliveira; Bergqvist, Lilian Paglarelli. 2002. Novos achados de Panochthus (Mammalia, Cingulata, Glyptodontoidea) no nordeste do Brasil. Revista Brasileira de Paleontologia, vol. 4, p. 51-62.

Porpino, Kleberson de Oliveira; Santos, Maria de F.C.F. dos; Bergqvist, Lilian Paglarelli. 2004. Registros de mamíferos fósseis no Lajedo de Soledade, Apodi, Rio Grande do Norte, Brasil. Revista Brasileira de Paleontologia, vol. 7, n 3, p. 349358.

Prado, J. L., Alberdi, M. T., Azanca, B., Sánchez, B. \& Frassinetti, D. 2005. The Pleistocene Gomphotheriidae (Proboscidea) from South America. Quaternary International, 126- 128: 21-30.

Prado, J. L. ; Alberdi, M. T. 2008. Restos de Hippidion y Equus (Amerhippus) procedentes de las Barrancas de San Lorenzo, Pleistoceno tardío (Provincia de Santa Fé, Argentina). [Hippidion and Equus (Amerhippus) from the San Lorenzo cliff, late Pleistocene (Santa Fe province, Argentina).] Revista Española de Paleontología, 23 (2), 225-236. ISSN 0213-6937.
Prevosti, F.J.; Vizcaíno, S.F. 2006. Paleoecology of the large carnivore guild from the late Pleistocene of Argentina. Acta Palaeontologica Polonica 51 (3): 407-422.

Ranzy, A. 2000. Paleoecologia da Amazônia - Megafauna do Pleistoceno. Florianópolis, Editora da UFSC, $101 \mathrm{p}$.

Ribeiro, Ricardo da Costa; Carvalho, Ismar de Souza, 2009. Megafauna do Quaternário tardio de Baixa Grande, Bahia, Brasil Anuário do Instituto de Geociências - UFRJ Vol. 32 - 2 / p. 42-50

Rincon, Ascanio D.; White, Richard S.; McDonald, Hugh Gregory. 2008. Late Pleistocene Cingulates (Mammalia, Xenarthra) from Mene de Inciarte tar pits, Sierra de Perijá, western Venezuela. Journal of Vertebrate Paleontology, vol. 28. $\mathrm{n}^{\circ} 1$, p. 197-207.

Silva, Fabiana Marinho da; Alves, Rosembergh da Silva; Barreto, Alcina Magnólia Franca; SÁ, Fabrício Bezerra de; LINS E SILVA, Ana Carolina Borges. 2006. A megafauna pleistocênica de Pernambuco. Estudos Geológicos, vol. 16, ${ }^{\circ}$ 2, p. 55-66.

Spix, J.B.; Martius, C. 1976. Viagem pelo Brasil: 1817-1820. São Paulo: Melhoramentos.

Sei, 1998. Superintendência de Estudos Econômicos e Sociais. Análise dos atributos climáticos do estado da Bahia. Salvador: (Série estudos e pesquisas, 38).

Sei, 2015. Superintendência De Estudos Econômicos e Sociais. Estado da Bahia. Salvador: 1 mapa, color. Escala 1:1.500.000. Disponível em:

$<$ http://www.sei.ba.gov.br/site/ge oambientais/mapas/pdf/bahia_ma pa_1v5m_2015_sei.pdf $>$.

Sei, 2018. Superintendência De Estudos Econômicos e Sociais. Perfil dos 
MEGAFAUNA PLEISTOCÊNICA DA MICRORREGIÃO DE SENHOR DO BONFIM...

Territórios de Identidade / Superintendência de Estudos Econômicos e Sociais da Bahia. -Salvador: 3 v. p.252 (Série territórios de identidade da Bahia, v. 3).

Zurita, Alfredo; Scillato-Yané, Gustavo J.; Carlini, Alfredo A. 2005.
Paleozoogeographic,

biostratigraphic, and systematic aspects of the Genus Sclerocalyptus Ameghino, 1891 (Xenarthra, Glyptodontidae) of Argentina. Journal of South American Earth Sciences, $n^{\circ} 20, \mathrm{p}$. 121-129. 